\title{
Sensitivity and stability: A signal propagation sweet spot in a sheet of recurrent centre crossing neurons
}

\author{
Christopher L. Buckley and Seth Bullock \\ Science and Engineering of Natural Systems (SENSe) group, School of Electronics and Computer Science, University of \\ Southampton, Southampton, SO17 1BJ, UK
}

\begin{abstract}
In this paper we demonstrate that signal propagation across a laminar sheet of recurrent neurons is maximised when two conditions are met. First, neurons must be in the so-called centre crossing configuration. Second, the network's topology and weights must be such that the network comprises strongly coupled nodes, yet lies within the weakly coupled regime. We develop tools from linear stability analysis with which to describe this regime, and use them to examine the apparent tension between the sensitivity and instability of centre crossing networks.
\end{abstract}

Key words:

Signal propagation, centre crossing networks, linear stability analysis, May-Wigner threshold, network dynamics

\section{Signal Propagation}

Signal propagation is central to the control systems of all adaptive agents in that it is crucial for the effective transduction of sensory input into motor output. Biological systems seem to achieve successful signal propagation over extended networks of neurons with relative ease (Carlson, 1991). Feedforward neural architectures have been employed to investigate how signals propagate across networks and can construct complex mappings between input and output (Rumelhart and McClelland, 1986). However, in general, biological neural networks are recurrent, even in systems that have previously been idealised as feed-forward in nature, e.g., the columns within the visual cortex have recurrent connections within and between layers (Carlson, 1991). Signal propagation across such recurrent networks is likely to be more complex than in feed-forward networks,

Email address: clb05r|sgb@ecs.soton.ac.uk (Christopher L. Buckley and Seth Bullock).

Preprint submitted to Elsevier where is taken for granted.

There has been a deal of speculation in neuroscience concerning mechanisms that could promote signal propagation across a sequence of neurons (Turrigiano, 1999). One set of ideas involves the behaviour of nodes that tend to interact at the centre of their operating ranges. In general, networks of such neurons are thought to be computationally rich. More specifically, in this regime, nodes are maximally sensitive to input, potentially facilitating more efficient signal propagation across extended networks. Moreover, Turrigiano (1999) describes how homeostatic processes (HPs) might actively "keep neurons at the centre of their operating ranges" (Turrigiano, 1999).

Inspired by this work, Williams (2006) studied how an abstraction of these HPs affected the ability of a continuous time recurrent neural network (CTRNN) to propagate signals. In this work, HP provided a simple feedback mechanism that altered the gain and bias of a node such that its input tended to lie at the centre of its transfer function. He hy- 
pothesized that networks composed of such nodes would be better able propagate signals, because local HP at the level of individual nodes would drive networks into the most sensitive region of their dynamics.

Williams found that HP drove systems toward a configuration that has been identified as significant within the CTRNN literature. In this so-called "centre crossing configuration" all nodes in a CTRNN interact at the centre of their sigmoid transfer functions (Mathayomchan and Beer, 2002), a mathematical property that bears close resemblance to the biological ideas highlighted by Turrigiano, amongst others. Williams also demonstrated that signal propagation was improved within such centre crossing networks.

However, this signal propagation was impoverished within larger networks, and did not approach the performance achieved by an equivalent feedforward architecture even for small networks (pers. comm.). One possible reason for these results can be induced from the original work on centre crossing CTRNNs (Mathayomchan and Beer, 2002). Here, it was demonstrated that the generation of rhythmic patterns evolved more readily in such networks. This is due to the fact that centre crossing networks are likely to produce oscillatory dynamics. Such oscillatory behaviour is likely to corrupt the transmission of signals across extended networks and explain why such networks would be outperformed by feed-forward networks that do not exhibit such autonomous oscillations.

There seems to be a tension between these two accounts of the utility of centre crossing networks. On the one hand, their sensitivity helps to prevent signal loss while, on the other, they are prone to interfering reverberant activity. Here, we attempt to resolve this conflict through the use of linear stability analysis and random matrix theory. We start by briefly outlining the theoretical tools that we employ before applying them to a simple model of signal propagation across a laminar CTRNN. First, we show that the May-Wigner threshold on system stability, originally derived for linear systems, constitutes a bound on the stability of nonlinear CTRNNs. Then we go on to demonstrate that signal propagation is maximised just below the May-Wigner threshold for a number of CTRNN topologies. We consider these results in relation to "centre crossing ideas" developed within research on both natural and artificial neural networks.

\section{Linear Stability Analysis}

In this section, we show that it is possible to obtain insights into the properties of an ensemble of nonlinear systems using linear stability analysis (LSA). Specifically, we claim that this type of analysis allows us to demarcate stable and unstable regions in the CTRNN parameter space. We start by demonstrating that the absolute strength of the coupling around all CTRNN equilibria is bound by their weights. We then present results that allow us to describe a stable region in the parameter space of linear networks with weights that are normally distributed. Finally, we combine these insights and argue that they allow us to numerically and analytically calculate a bound for the stability of CTRNNs.

\subsection{Coupling in a CTRNN at Equilibrium}

The CTRNN (Beer, 1995), or leaky integrator, equation for $N$ nodes is given by

$$
\dot{y}_{i}=-y_{i}+\tanh \left(\sum_{j=1}^{N} \omega_{i j} y_{j}+\theta_{i}+I_{i}\right)
$$

where $y_{i}$ represents activation at the $i^{\text {th }}$ neuron, $\omega_{i j}$ is the weight on the connection between neurons $i$ and $j$. The parameter $\theta_{i}$ is the bias value at the $i^{\text {th }}$ neuron. $I_{i}$ is the sensory input at node $i$ at time $t$ and is assumed to be zero unless specified. Note: in this work, for simplicity, we neglect the usual timescale parameter $\tau_{i}$. Hence each CTRNN is fully parameterized by the set of weights and biases $[\Omega, \Theta]$.

Analysis of the dynamics of these systems is difficult even in the two-node case, see Beer (1995). However progress can be made by examining the dynamics around such a system's equilibrium positions (Strogatz, 1994). In general, an $N$-node CTRNN will have multiple equilibria. Let us focus on the general case denoted by $\mathbf{y}^{*} \equiv\left(y_{1}^{*}, \ldots, y_{N}^{*}\right)$. It is possible to linearize this non-linear system around this equilibrium and study the dynamics infinitely close to it as described by the Jacobian (Strogatz, 1994; Murray, 1989), a matrix of interaction strengths which in this case is given by

$$
J=\left(\begin{array}{ccc}
\omega_{11}^{e f f}-1 & \ldots & \omega_{1 N}^{e f f} \\
\vdots & & \vdots \\
\omega_{N 1}^{e f f} & \ldots & \omega_{N N}^{e f f}-1
\end{array}\right)_{\mathbf{y}^{*}}
$$


where we have made the following substitutions

$$
\omega_{i j}^{e f f} \equiv \omega_{i j} \frac{d\left[\tanh \left(U_{i}\right)\right]}{d U_{i}}
$$

and

$$
U_{i}=\sum_{j=1}^{N} \omega_{i j} y_{j}+\theta_{i}
$$

The Jacobian now consists of a set of effective weights $\left(\Omega^{e f f}\right)$ that characterise a linear system that can, nevertheless, be considered to approximate the dynamics of the original non-linear system around its identified equilibrium, $\mathbf{y}^{*}$.

It can be shown that the stability of the system around $\mathbf{y}^{*}$ is completely determined by the eigenvalues of the Jacobian (Strogatz, 1994). Specifically, a system is said be stable (i.e., it will quickly return after small perturbations) if all the real parts of the eigenvalues of the Jacobian are negative. Conversely, it will be unstable (i.e., small perturbations from equilibrium will diverge away) if any of the real parts of the eigenvalues of the Jacobian are positive (Strogatz, 1994).

The Jacobian for this system, and hence its stability, is dependent on $\mathbf{y}^{*}, \Theta$ and $\Omega$, see equation (3) and (4). However, it is possible to determine an upper bound on the absolute values of the entries of the Jacobian in terms of only the weight values, $\Omega$. Specifically, the contribution of $\mathbf{y}^{*}$ and $\Theta$ to the Jacobian is constrained by the maximum value of the first derivative of the transfer function (in this case the hyperbolic tangent function). Now fig. 1 shows how a hyperbolic tangent function, and its first derivative, $\operatorname{sech}^{2}(x)$, vary with their arguments. The latter reaches a maximum value of unity when $x=0$ and tends towards zero on either side.

We can therefore deduce that the maximum absolute values of the effective weights will be achieved when this function evaluates to unity in which case they will be equal the original network weights, i.e.,

$$
\operatorname{Max}\left[\left|\omega_{i j}^{e f f}\right|\right]=\left|\omega_{i j}\right|
$$

As such, the maximum possible absolute magnitude of the coupling around any equilibrium in a nonlinear system with weights $\Omega$ is just equal to the weights themselves. Variation in the equilibrium position $\mathbf{y}^{*}$ and the biases $\Theta$ may only reduce the coupling from this value. Furthermore, it is centre crossing networks that achieve this maximum possible absolute coupling, since, for such networks, nodes interact at the centre of their transfer functions, where the derivative is maximal. We present a more de-
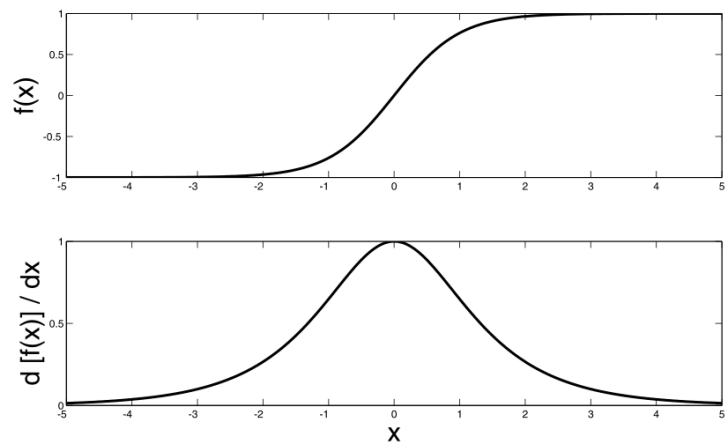

Fig. 1. The top panel shows a typical sigmoidal transfer function, here, the hyperbolic tangent. The bottom panel shows its first derivative, in this case $\operatorname{sech}^{2}(x)$. By definition, this derivative reaches a maximum coincident with the maximum gradient of the sigmoidal transfer function which, for any canonical transfer function, will occur for $x=0$.

tailed version of this argument elsewhere (Buckley, 2007).

\subsection{The May-Wigner Threshold}

In order to characterise general conditions for stability in CTRNNs, we will make use of work originally carried out by Gardner and Ashby (1970) and later formalised by May (1972). In a now classic study, Gardner and Ashby (1970) investigated stability criteria for large complex systems in terms of the effect of a network's size, connectivity and weight strength on its tendency to exhibit a stable point attractor. Gardner and Ashby considered linear networks of the following form

$$
\dot{y}_{i}=-y_{i}+\sum_{j=1}^{N} \omega_{i j} y_{j}
$$

They employed a numerical method of determining stability in networks of varying size, $N$, and connectivity, $C$. Non-diagonal entries of $\Omega$ were drawn from a normal distribution with zero mean and variance, $\alpha$. Note: all the self-weights (leading diagonal entries) were set to $\omega_{i i}=-1$, but the results here do not depend on this. Gardner and Ashby (1970) found that the probability of network stability, $p$, falls with increasing network size. Furthermore, it was observed that networks have a high probability of stability if either $\alpha$ or $C$ are low, and that this probability decreases with increasing $C$ or $\alpha$ (see fig. 2, solid line).

Later, May (1972) was able formalize these findings using analytical results from random matrix 


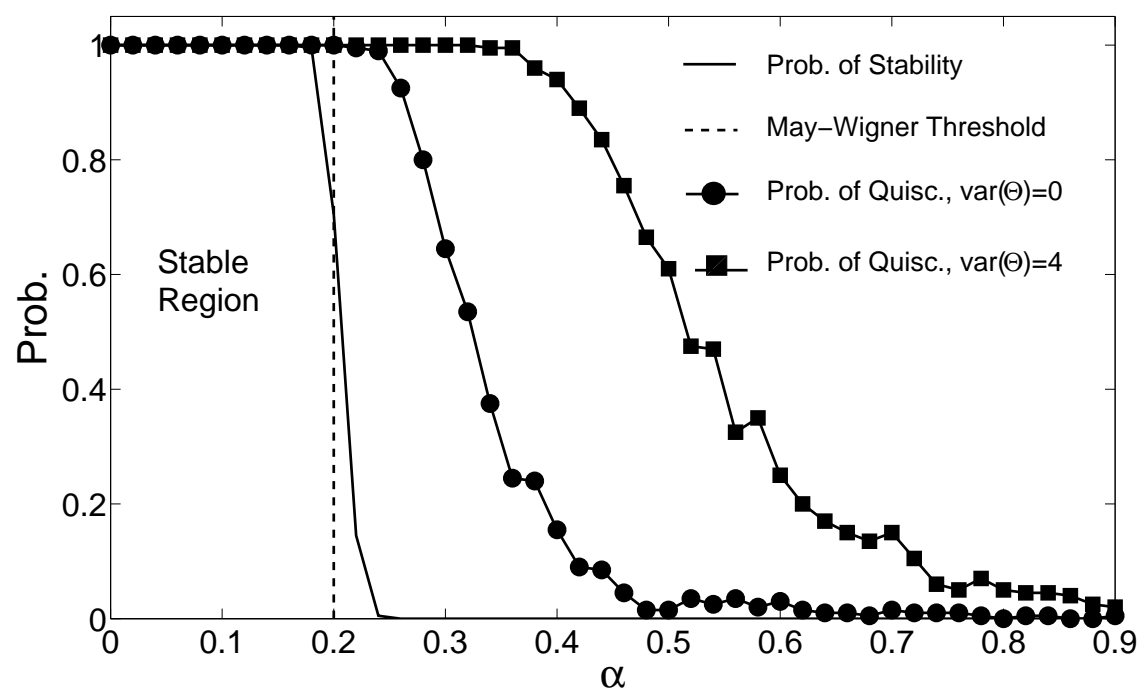

Fig. 2. Curves represent the impact of variance in a network's weight strengths, $\alpha$, on the probability of network stability for three different classes of network, with the vertical line indicating the prediction made by the May-Wigner threshold. First (solid line), probability of stability in linear networks comprising $N=100$ nodes of the kind studied by Gardner and Ashby (1970). Each network is connected according to a matrix populated with off-diagonal values drawn from a normal distribution, and leading diagonal values $\omega_{i i}=-1$. The probability of stability is determined by inspection of the numerically calculated eigenvalues of the Jacobian. Second (circles), fully connected networks of 100 unbiased CTRNN nodes (i.e., var $(\Theta)=0)$ with connection weights specified by the same matrices. Third (squares), the same sample of CTRNNs with node biases drawn from a normal distribution with zero mean and variance, $\operatorname{var}(\Theta)=4$. The proportion of networks that do not exhibit oscillatory behaviour is employed as a proxy for stability. Each data point is the average of 50 network realisations, and the error for all data points is less than $2 \%$.

theory (Wigner, 1959; Mehta, 1967). He was able to derive a critical threshold below which any network has a high probability of stability. Explicitly, he stated that in the limit of large system size $(N \gg$ 1) a system is almost certainly stable if

$$
N C \alpha^{2}<1
$$

This result, generally referred to as the MayWigner stability theorem, corresponds well with Gardner and Ashby's original findings and still holds as a very important threshold (Sinha and Sinha, 2005): a system is almost certainly stable if the variance of the weights conforms to the following inequality

$$
\alpha<\alpha_{M W} \equiv 1 / \sqrt{N C}
$$

Fig. 2 reconfirms the canonical result that the impact of variance in a network's weights on its probability of stability (as determined numerically for a sample of networks) agrees well with the analytically derived May-Wigner threshold. In order to make use of this powerful result, we will need to consider how it might be applied to the non-linear CTRNN.

\subsection{The Stability of CTRNNs}

Given the results of the last two sections, it is now possible to determine general conditions for the stability of CTRNNs with normally distributed weights. We will achieve this by using linear analysis tools to consider their (non-linear) behaviour around an arbitrary equilibrium, $\mathbf{y}^{*}$. Consider a CTRNN with $N$ nodes and $k$ connections per node. As before, each weight is drawn at random from a normal distribution with zero mean and variance $\alpha$. However, here, the network biases, $\Theta$, are similarly distributed normally with zero mean and variance $\operatorname{var}(\Theta)$.

While the May-Wigner threshold applies directly to systems of linear elements, the non-linear transfer functions of a CTRNN require us to employ the reasoning introduced in section 2.1. Since the absolute values of the effective weights associated with a particular equilibrium in a network's dynamics cannot be greater than that of their corresponding actual weights $\left(\omega_{i j}^{e f f}<\omega_{i j}\right)$, and these weights are normally distributed with zero mean, the variance 
of the effective weights cannot be greater than that of the actual weights $\left(\alpha^{e f f}<\alpha^{a c t}\right)$. Hence, if the variance of a network's actual weights lies below the May-Wigner threshold ( $\alpha<\alpha^{M W}$ ), so will the variance of its effective weights $\left(\alpha^{e f f}<\alpha^{M W}\right) .{ }^{1}$ Consequently, for any CTRNN where $\alpha<\alpha^{M W}$, each of its equilibria will have a high probability of stability.

The network simulation results presented in fig. 2 are consistent with this line of reasoning, suggesting that it is valid to co-opt these linear stability results in order to characterise the onset of instability in non-linear CTRNN systems. Here, networks of 100 nodes with weights and biases distributed as described above were forward integrated for 1000 time steps with a Euler step of $\delta t=0.05$. As an indication of instability in these networks, oscillatory behaviour is tested for by measuring the average deviation from the mean after a transient period of 500 time steps. Both for cases where nodes are unbiased $(\operatorname{var}(\Theta)=0$, circles) and where biases vary $(\operatorname{var}(\Theta)=0$, squares), no simulated network below the May-Wigner threshold exhibits oscillatory behaviour. Clearly, networks may be unstable in other ways, but the fact that we see no oscillatory behaviour below the threshold can be taken as reasonable evidence for the stability in that region. CTRNNs in this stable region bounded by the May-Wigner threshold can be considered to be examples of weakly coupled systems which are studied throughout neuroscience and are considered to be good models of the dynamics of networks of neurons in many parts of the nervous system (Hoppensteadt and Izhikevich, 1997).

\section{Signal Propagation in a Recurrent Sheet of CTRNN Nodes}

Here we examine signal propagation across laminar sheets of CTRNN nodes utilising the tools developed within the previous section. Each sheet consists of $N=60$ nodes arranged in a $L \times W=15 \times 4$ rectangular array. The networks are connected according to various topologies, see fig. 3. Each connection within the network (i.e., the value of each entry in the weight matrix, $\Omega$ ) is drawn from a normal distribution with zero mean and variance $\alpha$. Similarly, the biases of the network are again drawn from a normal distribution with zero mean and variance $\operatorname{var}(\Theta)$.

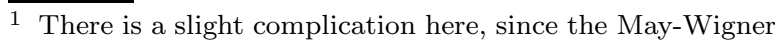
threshold does not speak to weight distributions with nonzero mean. See Buckley (2007).
}

(a)

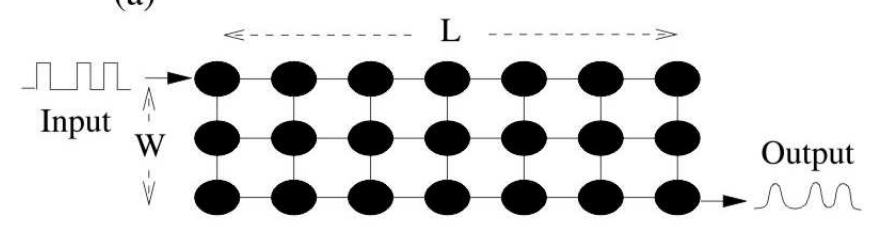

(b)

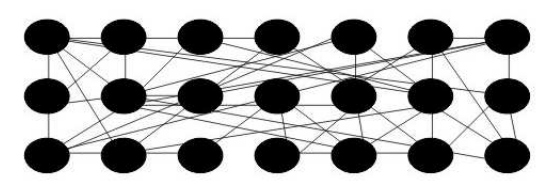

(c)

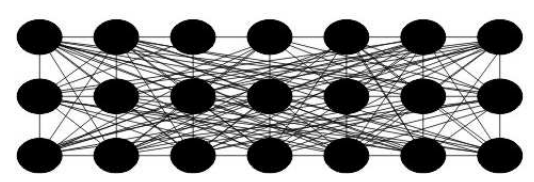

Fig. 3. A laminar sheet of $N$ CTRNN nodes arranged in an array with width, $W$, and length, $L$, is driven by a square wave input signal at one corner node. The correlation between this input signal and the output taken from the diametrically opposed node is measured for three different topologies: (a) a rectangular lattice, (b) the same lattice randomly rewired such that every node is assigned $k=4$ incoming edges at random, but out degree is free to vary, (c) a fully connected network.

All networks are forward integrated with an Euler step of $\delta=0.05$. Note: this way of randomly constructing CTRNNs resembles the way in which an initial population of neural networks is constructed prior to some period of artificial evolution, see, e.g., Beer (2003).

A square wave signal is applied to the input node $i=1$. This comprises intervals of low stimulation, $I_{1}=0$, for periods uniformly distributed over the interval $[50,400]$, and high stimulation, $I_{1}=1$, with length uniformly distributed over the interval $[50,200]$ time steps, see the top two panels of fig. 5 . We measure the correlation between the input signal and the output signal from the diametrically opposite node, see fig. 3. Note: calculating correlation involves scaling each signal by its variance and is therefore insensitive to the absolute magnitude of the signal. However, here we apply a small magnitude noise signal to each node $\left(\approx 10^{-6}\right)$ at every time step, which effectively masks any correlation between the input and extremely small output signals. Finally, the phase delay between input and output signal imposed by the shortest path length separating the input node from the output node is corrected for such that, for every measurement, the correlation is maximised, see the top left panel of 
fig. 5.

First we consider networks in which all bias values, $\Theta$, are set to zero. Note: this ensures that network equilibria occur where all node activations are zero. Furthermore, at such equilibria, all nodes interact at the centre of their sigmoidal transfer functions. Hence all such CTRNNs can be considered to be very simple examples of centre crossing networks.

Fig. 5 shows typical traces of the input, output and inter node activations for a lattice network (see fig. 3a). The two left-hand panels depict the dynamics of such a network parameterised to lie within the weakly coupled region below the May-Wigner threshold. The output signal closely maps the input with some consistent delay, but the absolute magnitudes of the node activations are very small, since the signal is significantly attenuated as it traverses the lattice. As a result, signal propagation performance is critically dependent on the scale of any noise within the system. For systems with small weight values, the output signal is so small that it is washed away by the internal noise injected at each node. The two right-hand panels depict the dynamics associated with a lattice parameterised to lie above the May-Wigner threshold. Networks in this region exhibit high magnitude complex dynamics unrelated to the input signal. In general the absolute value of the propagated signal increases with weight variance. Note the difference in scale on the $\mathrm{y}$-axes of the lower panels.

Fig. 4 shows how the correlation between input and ouput, $\operatorname{corr}$ (Input/Output), varies with the $\log$ of the variance of the weights, $\log _{10}(\alpha)$ for the three different network connection topologies given in fig. 3. The left-hand panel presents results for a lattice network (see fig. 3a), and shows that the correlation between input and output rises and then falls with the variance of the weights. More specifically, there is an intermediate region where the coupling between nodes is high enough to resist signal attenuation, but low enough to avoid instability. This "sweet spot" is located just below the May-Wigner threshold.

The right-hand panel of fig. 4 presents results for a rewired lattice (fig. 3b) and a fully connected network (fig. 3b). For these topologies, the short path length between input and output nodes ensures that the signal attenuation problem suffered by the lattice is not as significant. As a result, high correlation between input and output can be achieved with low weight variance. However, the figure confirms that signal propagation still falls sharply above the May-
Wigner threshold for these networks, despite the potential advantage conferred by their short minimum path lengths.

Note that the different topologies of the rewired lattice and fully connected network lead to differences between the results of both the numerically predicted probability of stability and the position of the analytically derived May-Wigner threshold. This fall in performance is well predicted both by the numerically calculated probability of stabillity and the analytically calculated May-Wigner threshold, further supporting the arguments made in section 2. Specifically, as the weight variance exceeds this threshold, reverberant oscillation and node saturation associated with the unstable regime destructively interferes with the transmission of information.

How do these results generalise to networks that are not in a centre crossing configuration? Fig. 6 shows how the input/output correlation varies with the $\log$ of the variance of the biases, $\log _{1} 0(\operatorname{var}(\Theta))$, for the three different network topologies. In each case, the variance of the weights, $\alpha$, is set according to fig. 4 such that it maximises signal propagation for unbiased networks. In all cases, increasing variance damages signal propagation. Nominally, this result is in line with Williams and Noble (2007).

Interestingly, effective signal propagation in both the fully connected network and the rewired lattice is more resistant to increasing variance in $\Theta$. This is likely to stem from the involvement of fewer nodes in the path along which the signal propagates. However, the key observation here is that departure from centre crossing configurations does damage signal propagation.

\section{Discussion and Conclusion}

Here we have suggested that not only is signal propagation across CTRNNs, and recurrent networks in general, maximised when they are in a centre crossing configuration, but that they must also lie within the weakly coupled regime bounded by the May-Wigner threshold. More accurately, while the May-Wigner threshold speaks to ensembles of networks with normally distributed weights, a more general stability criterion derived numerically via linear stability analysis provides a similar bound that can apply to networks in general. Furthermore, signal propagation is robust to internal noise to the extent that a networks nodes are strongly coupled. 

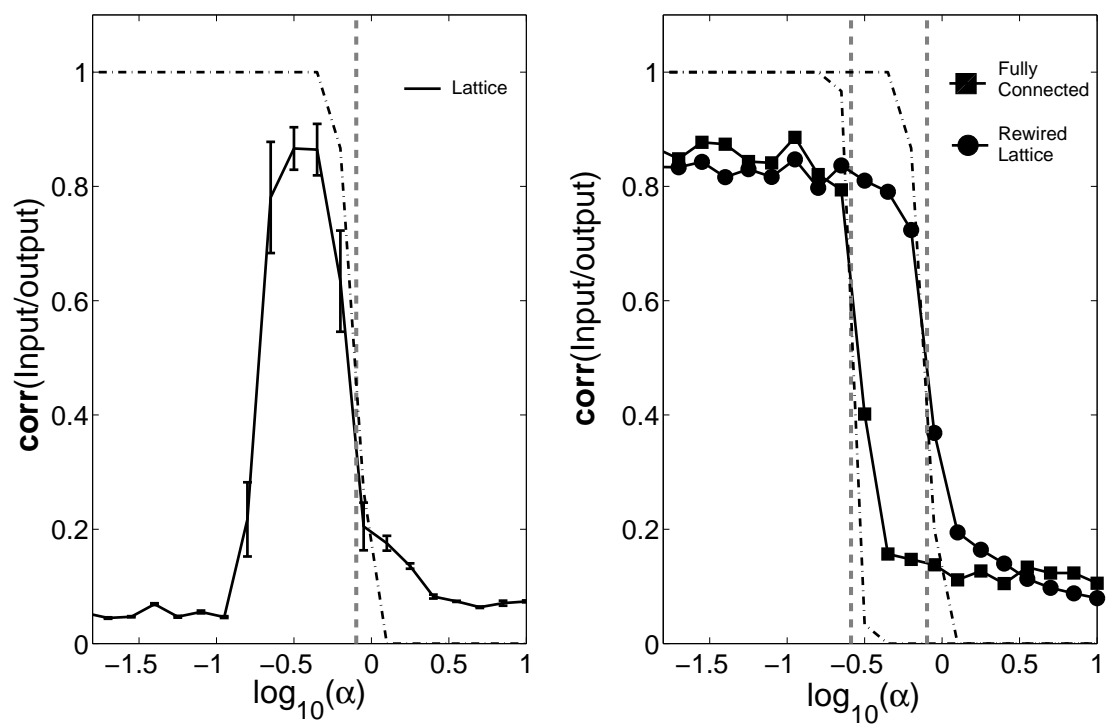

Fig. 4. The correlation between input and output signal, corr(Input/Output), versus the log of the variance of the weights, $\log _{10}(\alpha)$ for rectangular laminar networks with length $(L=15)$ and width $(W=4)$ and all biases, $\Theta$, set to zero. The solid line in the left-hand panel and the circles and squares in the right-hand panel show the correlation for a lattice network (see fig. 3a), randomly rewired lattice network (see fig. 3b) and fully connected network (see fig. 3c), repsectively. The dot-dashed lines are the respective numerically calculated probabilities of stability, and the vertical lines represent the analutically derived May-Wigner thresholds. Each data point is calculated as the average if 50 network realisations with the error-bars in the left-hand plot representative of standard deviations throughout.
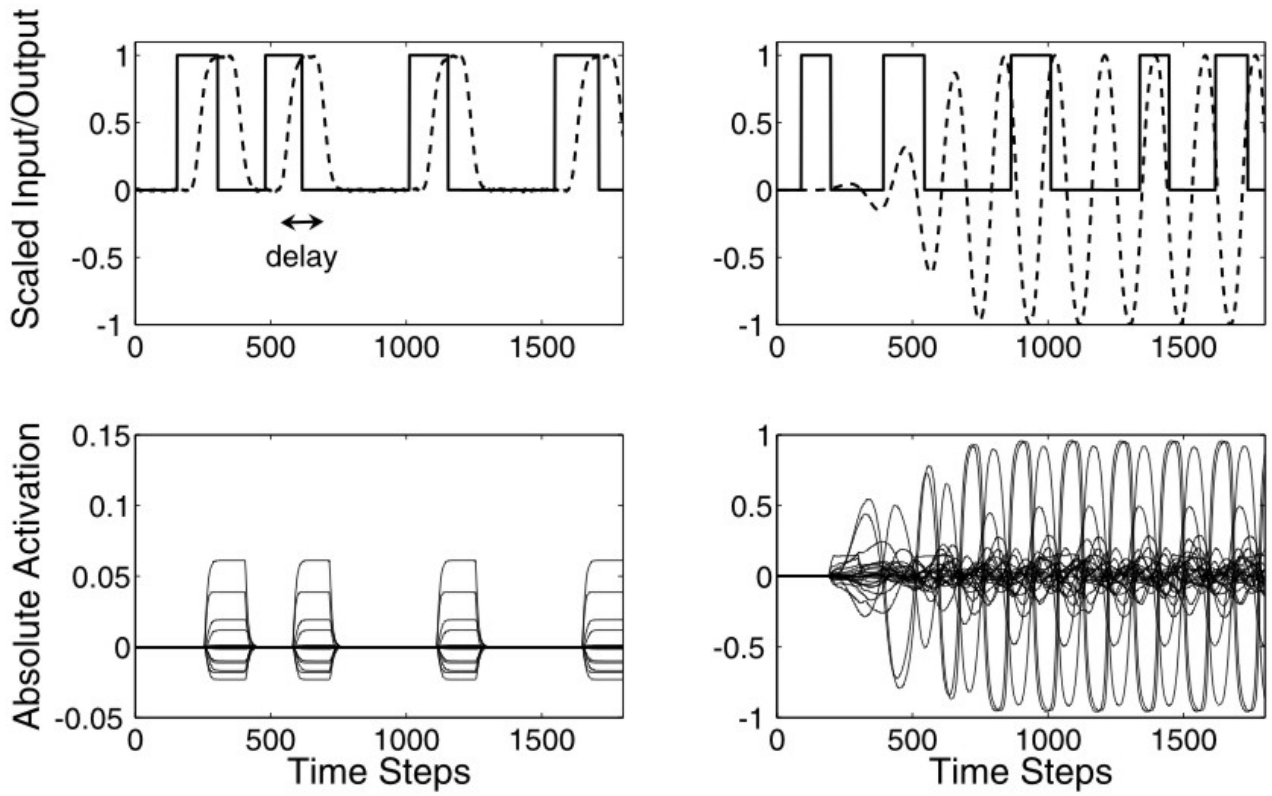

Fig. 5. Plots of network activity over time for the lattice network reported in figure 4 parameterised below the May-Wigner threshold (left-hand panels) and above it (right-hand panels). The two top panels show the input signal and the scaled output signal, solid and dashed lines respectively. The bottom two panels show a representative selection of the absolute activation values for all nodes. Note the difference in scale of $y$-axes on the bottom pair of graphs. The delay between the input and output signal is marked on the top-left panel. 


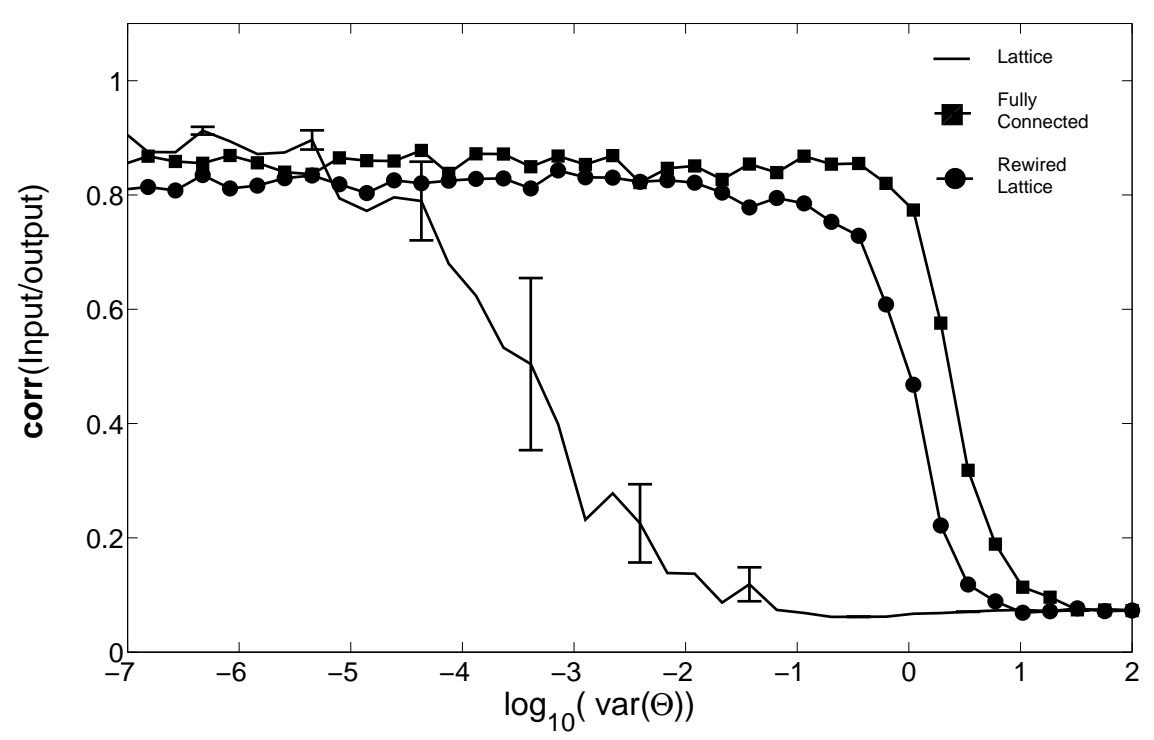

Fig. 6. The correlation between input and output signals, corr(Input/Output), versus the log of the variance of the biases, $\log _{10}(\Theta)$, for networks with length, $L=15$, and width, $W=4$, connected as a lattice (solid line), rewired lattice (circles) and fully connected (squares). All networks have weight variance, $\alpha$, which maximises the signal propagation across unbiased networks. Each data point is calculated as the average of 50 network realisations and representative standard deviations are given by the error bars on the solid line.

These two factors combine to ensure that a region just below the May-Wigner threshold is optimal for signal propagation in recurrent networks since it combines stability with low signal attenuation.

While it was apparent that network topologies resulting in short path lengths between input and output nodes (e.g., fully connected networks) achieved high performance in signal propagation, this performance was also bounded by the same thresholds on stability. In fact, since we are interested in signal propagation as a proxy for signal transduction, a requirement for the involvement of intermediate nodes that can provide a substrate for successive computational operations is implied, ruling out short path length as a solution to signal transduction in general.

These results are founded on the confirmation that the weak coupling region is well predicted by both the numerical calculations of linear stability analysis and also the analytical derivation of the May-Wigner threshold. That linear tools such as these can be used to make headway in understanding a class of non-linear network is encouraging, since there is real need to understand the dynamics of CTRNNs. While some models employ bio-inspired augmentation of CTRNN-style networks (Husbands et al., 1998; Williams, 2004), there is an increas- ing move in CTTRN research to treat them as arbitrary dynamical systems. Indeed, this is founded on the fact that they have been proven to be universal smooth function approximators (Funahashi and Nakamura, 1993).

However, it is one thing to demonstrate that a class of network is capable of arbitrary behaviour in general, and another to characterise the type of behaviour that such networks are liable, likely, or suitable to exhibit. Consequently, one interesting task is to characterise the parts of CTRNN parameter space that readily enable adaptive behaviour. In particular, how might particular bio-inspired mechanisms (e.g., homeostatic plasticity, neuromodulatory gases, etc.) be associated with regions within this space that scaffold generic dynamics conducive to certain tasks. The work presented here is intended as a contribution towards answering this style of question.

\section{References}

Beer, R. D., 1995. On the dynamics of small continuous-time recurrent neural networks. Adaptive Behavior 3 (4), 471-511.

Beer, R. D., 2003. The dynamics of active categorical perception in an evolved model agent (with com- 
mentary and response). Adaptive Behavior 4 (11), 209-243.

Buckley, C. L., 2007. A systemic analysis of the ideas imminent in neuromodulation. Ph.D. thesis, Electronics \& Computer Science, University of Southampton.

Carlson, N. R., 1991. Physiology of Behaviour: Fourth Edition. Allyn and Bacon.

Funahashi, K., Nakamura, Y., 1993. Approximation of dynamical systems by continuous time recurrent neural networks. Neural Networks 6, 801806.

Gardner, M. R., Ashby, W. R., 1970. Connectance of large dynamic (cybernetic) systems: Critical values for stability. Nature 228, 784-784.

Hoppensteadt, F. C., Izhikevich, E., 1997. Weakly Connected Neural Networks. Springer-Verlag, New-York.

Husbands, P., T. Smith, N. J., O'Shea, M., 1998. Better living through chemistry: Evolving gasnets for robot control. Connection Science 10, 185-210.

Mathayomchan, B., Beer, R. D., 2002. Centercrossing recurrent neural networks for the evolution of rhythmic behavior. Neural Computation 14, 2043-2051.

May, R. M., 1972. Will a large complex system be stable. Nature 238, 413-414.

Mehta, M. L., 1967. Random Matrices. Academic Press, New York.

Murray, J. D., 1989. Mathematical Biology. Springer, Heidelberg.

Rumelhart, D. E., McClelland, J. L., 1986. Parallel Distributed Processing: Explorations in the Microstructure of Cognition. MIT Press, Cambridge, MA.

Sinha, S., Sinha, S., 2005. Evidence of universality for the May-Wigner stability theorem for random networks with local dynamics. Phy. Rev. Let. E $71,1-4$.

Strogatz, S. H., 1994. Nonlinear Dynamics \& Chaos. Addison-Wesley, Reading MA.

Turrigiano, G. G., 1999. Homeostatic plasticity in neuronal networks: The more things change, the more they stay the same. Trends in Neuroscience 22, 221-227.

Wigner, E. P., 1959. Gruppentheorie und Ihre Anwendung auf die Quantenmechanik der Atomspektren, trans. J. J. Griffin. Academic Press, New York.

Williams, H., 2004. Homeostatic plasticity in recurrent neural networks. In: Schaal, S., Ijspeert, A., Billard, A., Vijayakumar, S., Hallam, J., Meyer,
J.-A. (Eds.), Eighth International Conference on the Simulation of Adaptive Behavior. MIT Press, Cambridge, MA, pp. 344-353.

Williams, H., Noble, J., 2007. Homeostatic plasticity improves signal propagation in continuous time recurrent neural networks. Biosystems 87 (2-3), 252-259.

Williams, H. P., 2006. Homeotstatic adaptive networks. Ph.D. thesis, Biosystems group, School of Computing, The university of Leeds. 